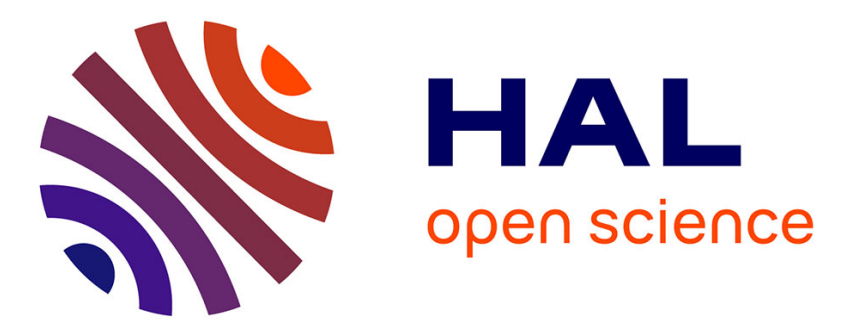

\title{
Lifetime estimation of moving vesicles in frequency-domain fluorescence lifetime imaging microscopy
}

Philippe Roudot, Charles Kervrann, François Waharte

\section{- To cite this version:}

Philippe Roudot, Charles Kervrann, François Waharte. Lifetime estimation of moving vesicles in frequency-domain fluorescence lifetime imaging microscopy. IEEE International Symposium on Biomedical Imaging: From Nano to Macro, ISBI 2012, May 2012, Barcelona, Spain. pp.668-671, 10.1109/ISBI.2012.6235636 . hal-00763790

\section{HAL Id: hal-00763790 \\ https://hal.inria.fr/hal-00763790}

Submitted on 11 Dec 2012

HAL is a multi-disciplinary open access archive for the deposit and dissemination of scientific research documents, whether they are published or not. The documents may come from teaching and research institutions in France or abroad, or from public or private research centers.
L'archive ouverte pluridisciplinaire HAL, est destinée au dépôt et à la diffusion de documents scientifiques de niveau recherche, publiés ou non, émanant des établissements d'enseignement et de recherche français ou étrangers, des laboratoires publics ou privés. 


\section{LIFETIME ESTIMATION OF MOVING VESICLES IN FREQUENCY-DOMAIN FLUORESCENCE LIFETIME IMAGING MICROSCOPY}

\author{
Philippe Roudot, Charles Kervrann \\ Inria Centre Rennes - Bretagne Atlantique \\ F-35042 Rennes
}

\begin{abstract}
We propose a framework for correcting the effect of vesicles motion in frequency domain FLIM imaging. Estimation of movement and lifetime are decoupled and alternatively performed. Robust M-estimation is involved to improve the accuracy of our estimate. Our method has been evaluated with both simulated and real samples.
\end{abstract}

Index Terms - FLIM, frequency-domain, phase modulation, particle tracking, template matching, M-estimator

\section{INTRODUCTION}

Fluorescence lifetime imaging microscopy (FLIM) is now a widely spread imaging technique for sensing fluorophore environment in a living biological sample (like $\mathrm{pH}$, ions...). Fluorescence lifetime (i.e. the average time a fluorophore stays in excited state before relaxing to its ground state possibly emitting a photon) is particularly useful to detect the Förster resonance energy transfer (FRET) which can allow to quantify spatial proximity between neighboring molecules [1]. Such in vivo techniques are known to be more precise than in vitro biochemical methods. Now the former gives a much better analysis of the global phenomenon than a sample-by-sample FLIM measure carried out by an operator. To overcome that problem automatization of FLIM sample analysis is required.

In comparison to the well-established time-correlated single-photon counting (TCSPC) technique, frequency-domain methods are known to be faster [2]. By using this technique Munster and Gadella [3] showed that photo-bleaching artifacts are easily negligible by permuting the frame recording order. Nevertheless, since it relies on intensity quantification over an image series, it is sensitive to sample movement that may lead to additional intensity variations [2]. To address this problem we propose a framework that exploits the intensity model of the frequency-domain FLIM output to jointly estimate trajectories and lifetimes of vesicles.

To our knowledge, no previous work have been done on this specific topic. Fourier transform or sine fitting is often recommanded in the case of static molecules $[4,5,3]$. However, a wealth of methods have already been proposed to track

\author{
François Waharte
}

\author{
UMR 144 CNRS - PICT-IBISA \\ F-75248 Paris
}

molecules in fluorescence imaging. Most of them rely on particle linking after spot detection in each frame [6]. Spot detection can be performed using Gaussian mixture model fitting [7], multi-scale wavelet analysis, top-hat filtering or local curvature analysis (see [8]). Linking is then achieved using scoring classification techniques, particle filtering or/and connectivity graph approach [7]. Other approaches also propose template cross-correlation [9] or Gaussian fitting [10]. The tracker of our method is inspired from this former technique.

In this paper we propose a statistical framework to address our specific problem. In a nutshell, our method tries to combine lifetime estimation and robust M-estimator tracking in a efficient and fast way. Estimation of movement and lifetime are decoupled and alternatively performed. Indeed, lifetime parameter estimation relies on an accurate estimation of vesicle locus, and the sinusoidal model that rules intensity variation temporally is exploited to enhance tracker performance. Spot detection is performed on the first frame. Actually, we do not need sophisticated spot detection scheme since our estimator is able to detect objects which are not conform with our model. This saves computations for applications.

The remainder of this paper is organized as follows: Section 2 briefly presents the physical process related to frequency-domain FLIM measurements. In Section 3, we present our procedure and we justify our vesicle template. We describe the procedure devoted to estimate movement and lifetime. Section 4 presents our promising results.

\section{FLUORESCENCE LIFETIME MEASUREMENT BY HOMODYNE DETECTION}

Frequency-domain FLIM images have a very specific intensity footprint which needs to be described for a better understanding of our approach. To that end, this Section briefly presents the theoretical basis for lifetime measurement in the frequency domain by homodyne detection (see [5] for a more detailed description).

Given a fluorescence sample excited with a single laser pulse, the impulse response $R(t)$ can be described by a sum of exponential function parameterized by decay times: these lifetimes represent the average time between its excitation 
time and return time to the ground state of each molecule present in the sample. For the sake of simplicity, we assume the impulse response to be described by a single exponential decay throughout the paper :

$$
R(t)=R_{0} e^{-\frac{t}{\tau}}
$$

with $R_{0}$ the intensity at time $t=0$ and $\tau$ the decay time.

Whereas the excitation source in time-domain methods is a laser pulse (like with TCPSC), the frequency-domain source is a sinusoidally modulated excitation $E(t)$ defined as:

$$
E(t)=C_{E}+A_{E} \sin \left(\omega_{E} t+\phi_{E}\right)
$$

where $\omega_{E}$ denotes the radial frequency of the excitation signal, $C_{E}$ the offset, $A_{E}$ the amplitude and $\Phi_{E}$ is the phase delay. Hence, the emitted fluorescent signal is defined as :

$$
\begin{aligned}
F(t) & =E(t) * I(t) \\
& =C_{F}+A_{F} \sin \left(\omega_{E} t+\phi_{E}-\arctan \left(\omega_{E} \tau\right)\right)
\end{aligned}
$$

where $*$ is the convolution operator. It turns out the measure of the phase delay $\phi_{E}-\arctan \left(\omega_{E} \tau\right)$ of $F(t)$ allows us to recover $\tau$. Nevertheless the experimental frequency in such experiment $(>1 \mathrm{MHz}$ ) is known to be too high to allow us to directly measure the phase when acquiring images. Therefore the signal is phase-modulated with $K \in \mathbf{N}$ sinusoidal signals $M_{k}(t)$ at the same frequency $\omega_{E}$ (homodyne detection) as :

$$
M_{k}(t)=C_{G}+A_{G} \sin \left(\omega_{E} t+\phi_{M_{0}}+\frac{2 \pi k}{K}\right), k \in[1, K]
$$

Thanks to the low pass effect of the CCD detector, the higher frequencies of the phase modulated signal $F(t) G_{k}(t)$ are negligible. Therefore we obtain $K$ time-independent signals described as a function of $k$ :

$$
\begin{aligned}
S_{\theta}(k) & =\mathcal{G}_{C C D}\left(F(t) G_{k}(t)\right) \\
& =C_{S}+A_{S} \cos \left(\frac{2 \pi k}{K}+\Phi_{\tau}\right), k \in[1, K]
\end{aligned}
$$

where $C_{S}=C_{F} C_{M}, A_{S}=A_{F} A_{M}, \Phi_{\tau}$ is the phase $\phi_{M_{0}}-$ $\phi_{E}+\arctan \left(\omega_{E} \tau\right)$ and $\theta=\left(C_{S}, A_{S}, \Phi_{\tau}\right)^{T}$. Now, the phase $\Phi_{\tau}$ can be retrieved using sine fitting or Fourier decomposition techniques thanks to the lower frequency of $S_{\theta}(k)$. The system phase delay $\phi_{G_{0}}-\phi_{E}$ is calibrated by recording a reference frame sequence of a fluorescent sample with known lifetime. Finally, as we monitor the frequency $\omega_{E}, \tau$ comes straightforwardly. Fig. 2 shows an example of lifetime computation using the Fourier transform on the stack presented in Fig. 1 with $K=12$. This example highlights the problem of moving vesicles which create unrealistic values.

\section{MEASURING LIFETIME IN LIVE-CELL IMAGING}

The method presented above has been successfully applied on immobilized samples [2]. However considering moving
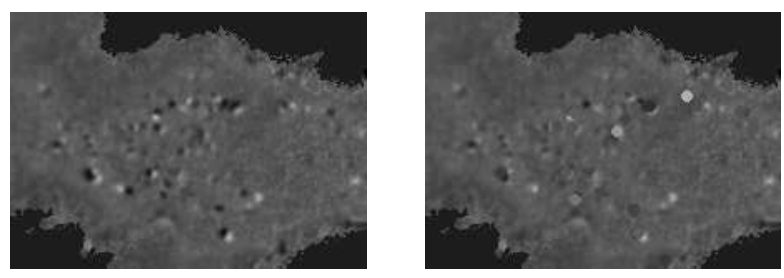

Fig. 2. On the left: Lifetime map using Fourier decomposition. Black and white spots are abberant values due to organels movement. On the right: reconstruction using our method.

vesicles is crucial when studying energy transfer. This study focus especially on moving sub-resolution vesicles. In this section we propose a model to describe the spatial and temporal variations of vesicles through the stack of $K$ frames. In the following we use time to refer to the order of phasemodulated frames, even if the order of phase-modulation can be chosen as pseudo-random [3] so that the order is not necessarily chronological. To alleviate complexity while taking advantage of parameter inter-correlation we experimented an iterative procedure where the lifetime parameter and the movement parameter are decoupled.

\subsection{Modeling spatial and temporal variations}

Several degradations were taken into account to model the vesicle intensity. In our approach the microscope point spread function (PSF), corresponding to a Bessel function, is approximated as a Gaussian function. Secondly, as the confocal equipment is not rigorously stigmatic the signal is composed by a background and a pinpoint vesicle before microscope diffraction. The background also follows the model (5) due to cytoplasmic fluorescence. Finally the background is assumed to be smooth over the PSF support. It comes the following expression for intensity on frame $k$ at position $x$ :

$$
I(k)(x)=S_{\theta_{b}}(k)(x)+S_{\theta_{0}}(k) \sigma_{\sigma}\left(x-x_{0}(k)\right)+\varepsilon(k)(x)
$$

where $S_{\theta_{0}}(k)$ is the vesicle mean intensity parameterized by $\theta_{0}=\left(C_{S_{0}}, A_{S_{0}}, \Phi_{\tau_{0}}\right)^{T}$ as in (5), $S_{\theta_{b}}(k)(x)$ is the background signal parameterized by $\theta_{b}(x)=\left(C_{S_{b}}(x), A_{S_{b}}(x), \Phi_{\tau_{b}}(x)\right)^{T}$, $\sigma_{\sigma}(x)$ is a Gaussian function of variance $\sigma^{2}$ and centered on the origin which describes the shape of the vesicle, $x_{0}(k)$ is the position of the vesicle on frame $k, \varepsilon$ is the noise introduced by the system. This set of parameters will be estimated in our procedure, though $\theta_{b}(x)$ needs to be estimated only on the vicinity of a vesicle.

\subsection{Iterated uncoupled parameter estimation}

This section presents the parameter estimation procedure leading to a robust estimation of the intensity decay time $\tau$. In a first step, we need to estimate the displacement of each individual vesicle. It turns out that movement estimation is 

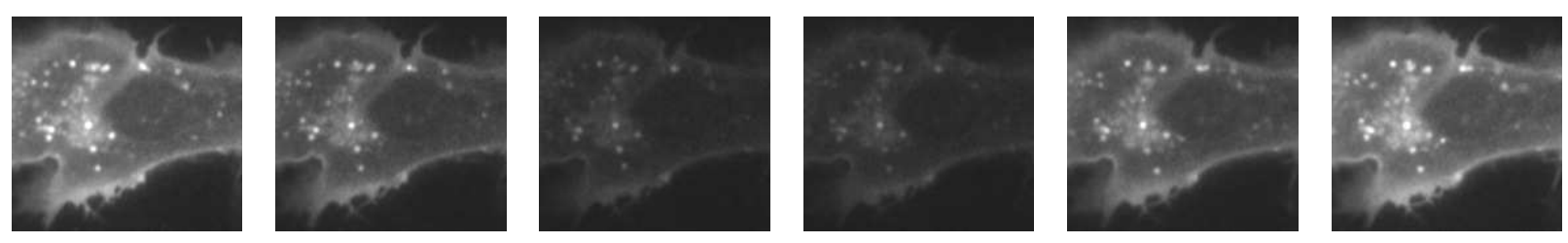

Fig. 1. An example of frequency-domain FLIM measurement with $K=6$ phase-modulation signal.

more efficient using a prediction given by the intensity model vesicles. Hence we propose a three steps procedure: sinusoid parameters prediction, robust tracking, lifetime estimation.

\subsubsection{Sinusoid parameter prediction}

We consider a small number of frame $K^{-} \in \mathbf{N}$ (we choose $K^{-}=3$ in samples where $K=12$ ) to predict $\theta_{0}$ and we consider a simpler model without the background influence:

$$
I(k)(x)=S_{\theta_{0}}(k) \sigma_{\sigma}\left(x-x_{0}(k)\right)+\varepsilon(k)(x), k \in\left[1, K^{-}\right]
$$

Vesicles are first tracked using a simple Gaussian fitting [10] over a square $7 \times 7$ window $W$. Before tracking, spot are detected using derivative of Gaussian filtering. This process provides us an estimate $x_{0}^{-}(k)$ and $\sigma^{-}$. Thus we can estimate $\theta_{0}$ by minimizing a robust error function:

$$
\begin{aligned}
& \theta_{0}^{-}=\underset{\theta_{0}}{\operatorname{argmin}} \\
& \sum_{\substack{k \in\left[1, K^{-}\right] \\
x \in W\left(x_{0}^{-}(k)\right)}} \rho_{\bullet}\left(I(k)(x)-S_{\theta_{0}}(k) \sigma_{\sigma^{-}}\left(x-x_{0}^{-}(k)\right)\right)
\end{aligned}
$$

where $W\left(x_{0}^{-}(k)\right)$ is a $7 \times 7$ window centered on the estimate $x_{0}^{-}(k)$. The function $\rho$ is an influence function chosen with a scale parameter couple $\bullet=(\lambda, \bar{\sigma})$ to weight down outliers during the minimization process. Experimentations show that the Leclerc influence function:

$$
\rho_{(\lambda, \bar{\sigma})}(r)=1+e^{-\frac{r^{2}}{\lambda \bar{\sigma}^{2}}}
$$

gives more stable results even if no significant differences are noticeable with other M-estimator (Tukey Bi-weighted or German-McClure among others). On each frame, variance of pseudo-residual is estimated using a robust least trimmed square estimator [11], the maximum is kept for $\bar{\sigma}^{2}$.

$C_{S_{0}}$, and $A_{S_{0}}$ are initialized using respectively the mean and the range of $\left\{\frac{I(x)(k)}{\sigma_{\sigma^{-}}\left(x-x_{0}^{-}(k)\right)} \mid k \in\left[1, K^{-}\right], x \in W\left(x_{0}^{-}\right)\right\}$ and $\Phi_{\tau_{0}}=\phi_{M_{0}}-\phi_{E}+\arctan \left(\omega_{E} \tau_{r e f}\right)$ with $\tau_{r e f}$ set to the fluophore lifetime measured without FRET (e.g. 2.5 ns for the EGFP presents in our experimental samples).

\subsubsection{Robust track}

Now $x_{0}(k)$ is estimated for each frame, taking advantage of $\theta_{0}^{-}$. Tracking is performed frame-by-frame using the same robust error function with different variables:

$$
\begin{aligned}
& \left(\tilde{x}_{0}(k), \tilde{\sigma}\right)=\underset{\left(\mathbf{x}_{0}(k), \sigma\right)}{\operatorname{argmin}} \\
& \sum_{x \in W\left(x_{0}(k)\right)} \rho_{\bullet}\left(I(k)(x)-S_{\theta_{0}^{-}}(k) \sigma_{\sigma}\left(x-x_{0}(k)\right)\right), k \in[1, K]
\end{aligned}
$$

As in Section 3.2.1 the most appropriate cost function is again the Leclerc estimator. Though the least median of square robust variance estimate [11]:

$$
\bar{\sigma}=1.4826 \operatorname{median}_{i \in\left[1, \mid W\left(\widetilde{x}_{0}(k) \mid\right]\right.}\left|r_{i}-\operatorname{median}_{i \in\left[1, \mid W\left(\widetilde{x}_{0}(k) \mid\right]\right.}\right| r_{i}||
$$

where $\left\{r_{i}\right\}$ are residuals of an ordinary least square estimation, performed better than least trimmed square estimator.

Initialization is performed with $\sigma=\sigma^{-}$and $x_{0}(k)=$ $\tilde{x}_{0}(k-1), x_{0}(0)=\tilde{x}_{0}(0)$ is determined by spot detection.

\subsubsection{Lifetime estimation}

In this step $\theta_{0}$ is estimated again taking advantage of our tracker estimate $\tilde{x}_{0}(k)$ for $k \in[1, K]$. As the measure must be as precise as possible, we do not neglect the influence of the background. Therefore we estimate $\theta_{b}(x)$ for $x \in \underset{k \in[1, K]}{\cup} W\left(\tilde{x}_{0}(k)\right)$ then subtract $S_{b}(k)(x)$ from the image. Background estimation relies on cytoplasmic autofluorescence described by $S_{b}(k)(x)$ in (5). Similarly to [12] we use an asymmetric M-estimator to discriminate background over vesicles. Though this last implementation does not handle still particles: if a vesicle does not move, there is no way to interpolate the underlying background. To that end we add the neighboring pixels influence in the M-estimation which gives the following robust error function to minimize:

$$
\begin{aligned}
& \tilde{\theta}_{b}(x)=\underset{\theta_{b}}{\operatorname{argmin}} \\
& \sum_{\substack{w \in W(x) \\
k \in[1, K]}} \rho_{\bullet}\left(I(k)(w)-\left(C_{S_{b}}(x)+A_{S_{b}}(x) \cos \left(\frac{2 \pi k}{K}+\Phi_{\tau_{b}}(x)\right)\right)\right), \\
& x \in \underset{\substack{k \in[1, K] \\
\cup}}{\cup} W\left(\tilde{x}_{0}(k)\right)
\end{aligned}
$$

where $W(x)$ is a $7 \times 7$ window centered on $x$. As in [12] a Leclerc estimator is chosen as influence function with $\bullet=$ $(\lambda, \sigma)$ chosen assymetricaly for positive and negative residuals. Over-weighting the negative residual disadvantage the 


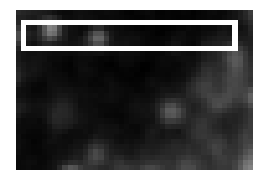

Sample

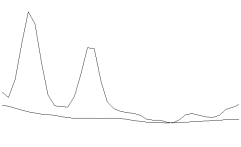

Profile

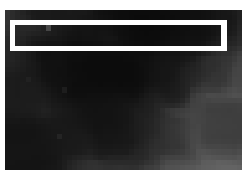

Background
Fig. 3. Background estimation on experimental sample, diagram shows both profile in highlighted region.

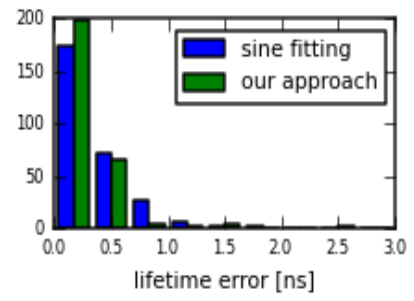

(a) Lifetime estimation method

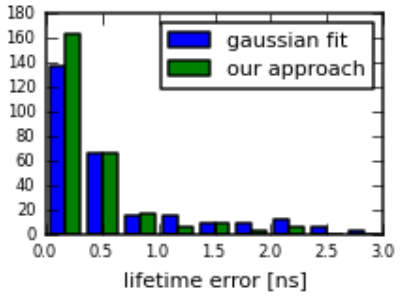

(b) Tracking method
Fig. 4. Comparing vesicles lifetime histograms on simulation.

pixel belonging to a spot, as the results $\theta_{b}$ describes the intensity of the background. Fig. 3 highlights the results.

After background subtraction, $\theta_{0}$ is estimated as in Section 3.2.1 over $K$ frames instead of $K^{-}$. It comes the parameter $\Phi_{\tau_{0}}$ and subsequently $\tau_{0}=\frac{\tan \left(\Phi_{\tau_{0}}-\phi_{M_{0}}+\phi_{E}\right)}{\omega_{E}}$. We could iterate on estimation of $x_{0}$ and $\theta_{0}$ though a single step already gives satisfying results.

\section{RESULTS AND DISCUSSION}

This section presents the results of our experimentation conducted on both synthetic and real sample.

Our simulations reproduce our model. Background is simulated with a real vesicle-less sample and cytoplasmic fluorescence is simulated with constant parameter $\theta_{b}$ on the background. Vesicles intensities follow (7) and movement is limited to a two pixels range. A mixed Gaussian noise is then applied to simulate measurement impact. Fig. 4(a) shows that our lifetime estimation method outperform the classic one on tracked vesicles (Section 3.2.1) while Fig. 4(b) compares our tracker with a basic Gaussian fitting (Section 3.2.2).

Uncontrolled vesicles interactions on experimental samples makes the generation of ground truth of lifetime impossible. Nevertheless we compared tracking accuracy. Track has been hand measured using I. Smal's MJtrack [8] on various confocal FLIM measurement samples provided courtesy of Institut Curie. Fig. 4 shows that tracking performance are lower on the darkest frames, preliminary investigations led us to focus on the noise model on those frames in future works. Fig. 2 shows an example of reconstruction.
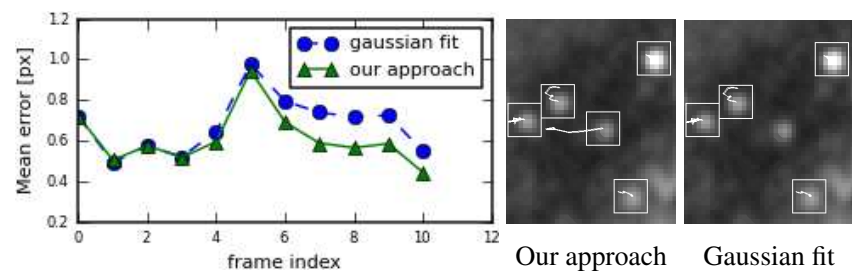

Fig. 5. Comparing tracks of 30 vesicles of a real sample.

\section{CONCLUSION}

Our method allows to retrieve lifetime of moving vesicle in an efficient and fast way on frequency-domain FLIM measurement. This study shows the importance of taking the intensity model into account while tracking vesicles. More tracker such as linking method will be quantitatively compared to assess the differences in performance of our procedure which is much less demanding in the spot detection area.

\section{REFERENCES}

[1] M.A. Hink, T. Bisseling, and A.J.W.G. Visser, "Imaging proteinprotein interactions in living cells," Plant molecular biology, vol. 50, no. 6, pp. 871-883, 2002.

[2] K. Suhling, P.M.W. French, and D. Phillips, "Time-resolved fluorescence microscopy," Photochemical \& Photobiological Sciences, vol. 4, no. 1, pp. 13-22, 2005.

[3] E.B. van Munster and T.W.J. Gadella Jr, "Suppression of photobleaching-induced artifacts in frequency-domain flim by permutation of the recording order," Cytometry Part A, vol. 58, no. 2, pp. 185-194, 2004

[4] BQ Spring and RM Clegg, "Image analysis for denoising fullfield frequency-domain fluorescence lifetime images," Journal of microscopy, vol. 235, no. 2, pp. 221-237, 2009.

[5] J.R. Lakowicz and B.R. Masters, "Principles of fluorescence spectroscopy," Journal of Biomedical Optics, vol. 13, pp. 029901, 2008.

[6] E. Meijering, I. Smal, and G. Danuser, "Tracking in molecular bioimaging," Signal Processing Magazine, IEEE, vol. 23, no. 3, pp. 46-53, 2006.

[7] D. Thomann, J. Dorn, PK Sorger, and G. Danuser, "Automatic fluorescent tag localization ii: improvement in super-resolution by relative tracking," Journal of microscopy, vol. 211, no. 3, pp. 230-248, 2003.

[8] I. Smal, M. Loog, W. Niessen, and E. Meijering, "Quantitative comparison of spot detection methods in fluorescence microscopy," Medical Imaging, IEEE Transactions on, vol. 29, no. 2, pp. 282-301, 2010.

[9] S. Bonneau, L. Cohen, and M. Dahan, "A multiple target approach for single quantum dot tracking," in Biomedical Imaging: Nano to Macro, 2004. IEEE International Symposium on. IEEE, 2004, pp. 664-667.

[10] C.M. Anderson, G.N. Georgiou, IE Morrison, GV Stevenson, and R.J. Cherry, "Tracking of cell surface receptors by fluorescence digital imaging microscopy using a charge-coupled device camera. lowdensity lipoprotein and influenza virus receptor mobility at 4 degrees c," Journal of Cell Science, vol. 101, no. 2, pp. 415, 1992.

[11] P.J. Rousseeuw, A.M. Leroy, and J. Wiley, Robust regression and outlier detection, vol. 3, Wiley Online Library, 1987.

[12] J. Boulanger, C. Kervrann, and P. Bouthemy, "Estimation of dynamic background for fluorescence video-microscopy," in Image Processing, 2006 IEEE International Conference on. IEEE, 2006, pp. 2509-2512. 